\title{
An Economic Analysis of Exclusion Restrictions for Instrumental Variable Estimation
}

\author{
Gerard J. van den Berg *
}

October 21, 2005, preliminary

\begin{abstract}
Instrumental variable estimation requires untestable exclusion restrictions. With policy effects on individual outcomes, there is typically a time interval between the moment the agent realizes that he may be exposed to the policy and the actual exposure. In such cases there is an incentive for the agent to acquire information on the value of the IV. This leads to violation of the exclusion restriction. We analyze this in a dynamic economic model framework. This provides a foundation of exclusion restrictions in terms of economic behavior that takes costs and benefits into account. The results are used to describe the policy evaluation settings in which instrumental variables are likely or unlikely to make sense. For the latter cases we analyze the asymptotic bias. The exclusion restriction is more likely to be violated if the outcome of interest strongly depends on interactions between the agent's effort before the outcome is realized and the agent's treatment status. The bias has the same sign as this interaction effect. Violation is not closely related to the weakness of the candidate instrument or to the size of the average treatment effect. In case of a social experiment, violation is more likely if the treatment and control groups are to be of similar size.
\end{abstract}

${ }^{*}$ Department of Economics, Free University Amsterdam, IFAU-Uppsala, IZA, IFS, and CEPR.

Keywords: treatment, policy evaluation, information, selection effects, randomization.

Thanks to Jim Heckman, Geert Ridder, and participants in the 2005 Econometric Society World Congress (London), for useful suggestions. 


\section{Introduction}

Instrumental variable estimation has since long been a standard econometric technique for dealing with endogeneity and selection issues in general, and for non-experimental policy evaluation in particular (see e.g. Angrist, Imbens and Rubin, 1996, Heckman, LaLonde and Smith, 1999, and Blundell and MaCurdy, 1999, for surveys). Basically, if one is interested in the effect of a "treatment variable" on an outcome variable, and the treatment is not exogenously assigned, then one may perform causal inference by exploiting the presence of variables that causally affect the treatment status but do not have a direct causal effect on the outcome. The latter restriction is called an exclusion restriction. Exclusion restrictions are identifying restrictions, so they can not be tested. This means that empirical results critically depend on the validity of the exclusion restriction, and that this restriction needs to be justified on a priori grounds.

With policy effects on individual outcomes, there is typically a time interval between the moment the agent realizes that he may be exposed to the policy and the actual exposure. For example, unemployed workers are aware of the existence of policies leading to treatments at some point of time in the future. As long as the instrumental variable affecting the treatment does not have a causal effect on the individual's behavior, the exclusion restriction is not violated. Often, a sufficient condition for this is that the agent does not observe the value of the instrumental variable. However, there is an incentive for the agent to acquire information on this value. After all, the probability of exposure to treatment is a determinant of the optimal strategy, and the more the agent knows about it, the better he can fine-tune his behavior in response to this, and the higher his expected present value will be. The agent's strategy affects the outcome of interest. Thus, the acquisition of the value of the variable that is used by the econometrician as instrumental variable leads to violation of the exclusion restriction and to incorrect empirical inference. ${ }^{1}$

In this paper we investigate, in the context of a dynamic economic framework, under which conditions it is optimal for the agent to acquire the value of the intended instrumental variable. This provides a foundation of exclusion restrictions in terms of economic behavior that takes costs and benefits into account. The results are used to describe the policy evaluation settings in which instrumental

\footnotetext{
${ }^{1}$ Earlier studies mentioning similar arguments, tacitly assuming that acquisition is free, include Abbring and Van den Berg (2003). For a very recent exposition, see Heckman and Navarro (2005). Note that we are not concerned with mechanical program lock-in effects that may affect the outcomes of participants before the end of the actual treatment participation.
} 
variables are likely or unlikely to make sense. This is especially useful since by definition no empirical evidence is available on the validity of exclusion restrictions.

At first sight one may think that information acquisition does not take place if and only if the acquisition costs are high, and that therefore the conclusion is simply going to be that instrumental variables estimation is particularly useful to study policy effects for agents with scarce resources. For active labor market policy analysis this would mean that it is particularly useful for agents at the bottom of the labor market, which coincides with the target group of most of these policy measures. However, this line of reasoning ignores the role of the value of the information that is acquired. We show that this leads to a different set of conclusions. The results point at the importance of the extent to which the treatment status and the agent's effort interact in the outcome.

The literature on instrumental variable estimation has recently been concerned with the use of so-called weak instruments, i.e. instrumental variables that are only weakly related to the treatment status (see e.g. Stock, Wright and Yogo, 2002, for a survey). Underlying this is the idea that weak instruments are less likely to be used by agents as direct causal inputs into the outcome of interest. We argue that in many cases this line of reasoning is incorrect, and therefore the attractiveness of weak instruments may be exaggerated.

We also use our framework to analyze how individuals may selectively affect their treatment status, and, more specifically, whether they selectively choose to become non-compliers, if they can acquire information on determinants of the treatment assignment process. We also consider model frameworks in which treatments are exogenous except for the possibility that individuals may decide to acquire information on the idiosyncratic determinants of the outcome and use this to affect their treatment status (leading to endogeneity of the latter). These models resemble the non-additive models examined by Imbens and Newey (2002). We examine how acquisition affects the OLS-type estimates of the treatment effect.

Throughout the paper we discuss two leading examples. In the first, the treatment is participation in a job search assistance program by an unemployed individual, and the policy intensity is different across two otherwise identical geographical regions. For example, in one region, the budget for the program per potential participant may be larger, so that the individual probability of being treated is larger, holding everything else constant. An individual may not be aware of the variation in policy intensities, or he may be aware of the distribution of intensities but not know his personally relevant intensity, in which 
cases a regional dummy indicator may be a valid instrumental variable. If the individual acquires his own region's policy intensity parameter then he may have an incentive to use this information before the treatment is realized. For example, it may be optimal to reduce the job search effort more if the treatment probability is large, because it is cheaper to provide effort after the treatment.

In the second example, we examine a double-blind experiment of say a medication to treat a disease. The randomized intention to treat then equals the treatment status, and this is unobserved until the outcome is observed. However, in the case of life-threatening diseases, an individual has an incentive to find out whether he receives medication or a placebo, for example by sending one tablet to a laboratory. If he discovers that he receives a placebo then he may choose a different lifestyle, which in turn affects the outcome. Alternatively, he may drop out and apply for participation in another medical experiment. Yet another option is to share the tablets among participating individuals. See e.g. Collins and Pinch (1998) and Schuklenk (2003) for examples concerning experiments of AIDS medication.

We mostly restrict attention to a binary instrument and a binary treatment status. We occasionally illustrate the analysis by examining unemployed job searchers who may participate in a job search assistance program. However, the results can be applied to many other situations, and indeed they can be straightforwardly applied to situations in which the treatment variable is not a policy variable. Also, the costs of information should be taken to cover not only monetary costs but also other types of effort. The paper is organized as follows: Section 2 presents the model framework, Section 3 derives the results concerning information acquisition, Section 4 discusses the implications for instrumental variable estimation, and Section 5 concludes.

\section{The model framework}

The main insights and results can be derived in a simple model framework with three time periods.

Consider an assignment process leading to the actual treatment status of an agent or decision maker. ${ }^{2}$ We assume that this process depends on a variable $Z$. At the onset of the first period, $Z$ is realized separately for each agent. We take the distribution of $Z$ as exogenously given and assume that $Z$ is dispersed, so $\operatorname{var}(Z)>0$. Clearly, $Z$ is the candidate instrumental variable. Each agent knows

\footnotetext{
${ }^{2}$ The model can be expressed in terms of counterfactual outcomes; see Angrist, Imbens and Rubin, 1996, for an exposition.
} 
the distribution of $Z$ across all agents. However, an agent does not necessarily know his own realization of $Z$. More precisely, in Period 1, each agent decides whether to acquire information on $Z$ at cost gamma $\gamma>0$ or not.

At the onset of Period 2, the agent determines his optimal strategy or effort s. The agent may or may not know his private value of $Z$ when determining $s$, but we assume that in Period 2 the agent does not know yet his treatment status $Y$. Without this assumption the analysis would be irrelevant, because there would never be any incentive to acquire information on the policy intensity. As we shall see, the analysis can allow for additional time periods and for agents to modify their strategy upon learning their value of $Y$, as long as their behavior before learning $Y$ has an effect on the outcome. The effort $s$ involves costs $c(s)$ to be paid in Period 2. These may be monetary costs or monetary equivalents of non-monetary costs.

In Period 3, the agent's actual treatment status $Y$ is realized. Both $s$ and $Y$ affect the outcome $U$, which is also realized in Period 3, simultaneously with or after the realization of $Y$. We express the outcome $U$ given $Y=y$ and given $s$ as $W \cdot f(y, s)+\varepsilon$, with $0 \leq f(y, s)$ and $0<W<\infty$. Here $W$ is just a multiplicative constant in the outcomes, and we merely introduce it to facilitate the analysis of effects of multiplicative changes in the outcomes. In the first leading example, $f$ may be the probability of making an income gain, and $W$ may be the expected income gain. The term $\varepsilon$ is an idiosyncratic outcome component. We assume that $\mathbb{E}(\varepsilon \mid Z)=0$, but all other determinants of $Y$ may be correlated to $\varepsilon$. The latter dependence captures the endogeneity of the actual treatment.

Summarizing, the sequence of events is as follows: the treatment assignment intensity $Z$ is realized, $Z$ is acquired or not, the effort $s$ is chosen, the treatment $Y$ is realized, and the outcome $W f(y, s)+\varepsilon$ is realized.

The above framework gives rise to a "reduced form" treatment evaluation model. First, as will become clear below, we may assume without loss of generality that $\mathbb{E}(Y \mid Z=z)=z$. This leads to a "treatment equation",

$$
Y=Z+\omega
$$

where $\mathbb{E}(\omega \mid Z)=0$. Secondly, we have an "outcome equation",

$$
U=W f(Y, s)+\varepsilon
$$

with $\mathbb{E}(\varepsilon \mid Z)=0$. The analyst observes $U, Y$, and $Z$.

Suppose that, more general than equation (1), we would specify $\mathbb{E}(Y \mid Z=$ $z)=g(z)$ for some function $g$. Typically, it is not difficult to estimate $g$, and 
this is why we simply redefine $g(z)$ as our $z$. This presupposes that $g$ varies with $z$. In other words, $Z$ as a candidate instrumental variable must be informative. Also, note that the specification $Y=Z+\omega$ with $\mathbb{E}(\omega \mid Z)=0$ can also capture discrete $Y$. Notably, if $Y$ is binary, one may define $Y=1 \Longleftrightarrow Y^{*}>0$ with $Y^{*}=Z+\omega^{*}$ and $\omega^{*}$ being uniformly distributed on the interval $[-1,0]$.

Suppose that in Period 1 the agent does not acquire his personal realization of $Z$. Then $Z$ only affects $U$ by way of $Y$, so $Z$ is a valid instrumental variable (IV) because the corresponding exclusion restriction (ER) is satisfied. Now suppose that in Period 1 the agent does acquire his personal realization of $Z$. Then $Z$ may affect his value of $s$. In that case, from equation (2), there is a causal effect of $Z$ on the outcome, resulting in a violation of the ER needed for instrumental variable estimation (IVE). Before we analyze this, we first derive in the next subsection the agent's optimal behavior concerning $s$ and concerning acquisition of $Z$.

\section{Economic behavior}

\subsection{Present values}

An agent maximizes his expected present value. To focus on the main issue we consider risk neutral agents. If the agent does not know his value of $Z$ then the expected present value $R_{0}$ at the onset of Period 2 equals

$$
R_{0}=\max _{s \in \mathcal{S}}-c(s)+\frac{1}{1+r} \mathbb{E}_{Z} \mathbb{E}_{Y \mid Z}(W \cdot f(Y, s))
$$

$\mathcal{S}$ denotes the choice set of $s$. We denote the optimal $s$ by $s_{0}$.

Now suppose that the agent knows that his value of $Z$ is $z$. The expected present value $R(z)$ at the onset of Period 2 is

$$
R(z)=\max _{s \in \mathcal{S}}-c(s)+\frac{1}{1+r} \mathbb{E}_{Y \mid Z=z}(W \cdot f(Y, s)) \quad(i=1,2)
$$

where $r$ is the discount factor. The optimal $s$ can be expressed as $s(z)$.

The value of information $V$ in Period 1 equals

$$
V=\frac{1}{1+r}\left(\mathbb{E}_{Z} R(Z)-R_{0}\right)
$$

It is optimal to acquire $Z$ in Period 1 if and only if $V>\gamma$. Clearly, the central issue of the paper is under which conditions this occurs. For the moment, we simplify the above expressions by subsuming the parameter $W /(1+r)$ into the function $f$, and $1+r$ in (5) into $V$. 
The first insight is that if $f$ is additive in $Y$ and $s$ then the optimal $s(z)$ in (4) does not depend on $z$, and it is equal to $s_{0}$. Consequently, $V=0$, and the agent does not acquire $Z$. In sum,

Proposition 1. If the outcome is additive in the treatment status and the optimal effort of the agent then the exclusion restriction is satisfied.

Throughout the paper we consider functions $f$ that are positive and increasing. As will become clear below, the main distinction in the derivations will be whether $\mathcal{S}$ is discrete or not. In the continuous case we often assume that $c$ is quadratic, with $c(s)=\frac{1}{2} c_{0} s^{2}$ and $c_{0}>0$. Also, many results will be derived for the following functional form for $f$,

$$
f(Y, s)=\psi_{0}+\psi_{1} s+\psi_{2} Y+\rho Y s
$$

with suitable restrictions on $\psi_{1}, \psi_{2}, \psi_{3}, \rho$ guaranteeing that $f$ is positive and increasing in the relevant intervals for $Y$ and $s$. We do however also provide results for non-parametric specifications of $f$. The functional form in (6) is concise and allows for explicit expressions and results for the quantities of interest. The interaction parameter $\rho$ captures the degree of complementarity $(\rho>0)$ or substitutability $(\rho<0)$ of treatment and effort, in the outcome.

The functional form (6) is less restrictive as may seem. First of all, with binary $s$ and $Y,(6)$ is non-parametric because the four possible values of $f$ $(f(1,1), f(1,0), f(0,1)$, and $f(0,0))$ are represented by $\psi_{0}, \psi_{1}, \psi_{2}$, and $\rho$. Secondly, as will become clear below, we may generalize the term $\psi_{0}+\psi_{2} Y$ at no cost to a general function $k_{2}(Y)$ (provided that the resulting $f$ is positive and increasing). So all results based on (6) generalize in this respect. The same applies if we replace $\psi_{1} s$ by a function $k_{1}(s)$, provided that $k_{1}(s)-c(s)$ is quadratic (and again $f$ is positive and increasing). The latter requirement is merely needed to ensure explicit expressions for the optimal $s$. In general, the right-hand side of (6) can be interpreted as the first part of an expansion of the underlying $f$. It is also useful to point out that in the related literature on decision making with a noisy signal about the unknown state of the world, the general effect of the shape of the outcome function $(f)$ on the value of information $(V)$ is typically too hard to analyze in terms of the model primitives, if no parametric assumptions are made on $f$ (see Persico, 2000, and Athey and Levin, 2001). We return to this literature below. 


\subsection{Optimal behavior with continuous effort}

Suppose that the choice set $\mathcal{S}$ of effort $s$ is an interval. We do not restrict $Z$ or $Y$ to be discrete or continuous, so the results below are valid in both cases.

We start with the model in which the functions $f$ and $c$ satisfy (6) and the quadratic specification $c(s)=\frac{1}{2} c_{0} s^{2}$, respectively. Within this framework we first consider the case $\rho>0$. This means that treatment and effort are complements in the outcome. The requirement that $f$ is positive and increasing then amount to the restrictions that $\psi_{0}>0, \psi_{1} \geq 0, \psi_{2} \geq 0$. Also, we assume that $c_{0}>0$. The optimal $s$ is always positive, and we assume that the lower and upper boundary of $\mathcal{S}$ are not binding for the optimal $s$.

Let $\bar{z}:=\mathbb{E}(Z)$ denote the population mean of $Z$. It is easy to derive that the optimal effort equals

$$
\begin{aligned}
s(z) & =\frac{\psi_{1}+\rho z}{c_{0}} \\
s_{0} & =\frac{\psi_{1}+\rho \bar{z}}{c_{0}}
\end{aligned}
$$

Note that $s(z)$ increases in $z$. This was to be expected. The complementarity of $f$ implies that the marginal return of effort is higher if the expected (beneficial) treatment level is higher. Also, the optimal effort is higher if the cost of it is lower, if the marginal return of it is higher, and if the degree of complementarity is higher. Note that the optimal $s$ does not depend on the marginal effect $\psi_{2}$ of the treatment $Y$.

By substituting (7) into equations (4) and (3), we obtain that

$$
V=\frac{1}{2 c_{0}} \rho^{2} \operatorname{var}(Z)
$$

Consequently, the ER is violated iff $\frac{1}{2 c_{0}} \rho^{2} \operatorname{var}(Z)>\gamma$.

Before we interpret this result we first analyze the case $\rho<0$. This means that treatment and effort are substitutes in the outcome. In the case of participation in active labor market programs, this case may be more realistic than the case $\rho<0$. For example, there may be an upper bound on the outcome, and the effort has to compete with efforts for other activities outside of the model. ${ }^{3}$ We also assume again that $c_{0}>0$. The requirement that $f$ is positive and increasing now leads to more complex restrictions on the parameters of $f$. For a start, we

\footnotetext{
${ }^{3}$ This does not rule out that the treatment and the efforts after the realization of the treatment are complements in their effect on outcomes in subsequent time periods. These are not in the present model but one can extend it to include them.
} 
take $\psi_{0}>0, \psi_{1}>0, \psi_{2}>0$. Next, we ensure that $f$ increases in $s$ for every $Y$. Sufficient for this is that $\operatorname{Pr}\left(Y<\frac{\psi_{1}}{-\rho} \mid Z\right)=1$, because together with the requirement that $\mathbb{E}(Y \mid Z=z)=z$ this implies that $\operatorname{Pr}\left(Z<\frac{\psi_{1}}{-\rho}\right)=1$, which implies the desired property of $f$. Note that it also implies that the optimal $s$ is positive. Finally, we ensure that $f$ increases in $Y$ for every $s$. This is satisfied if $s<\frac{\psi_{2}}{-\rho}$. The optimal $s$ decreases in $z$. The largest possible value of $s$ as a function of $z$ is therefore achieved at $z=0$, and this value equals $\psi_{1} / c_{0}$. Thus, $f$ has the desired property if $\psi_{1} / c_{0}<\psi_{2} /(-\rho)$ or, equivalently, $\psi_{2} c_{0}+\rho \psi_{1}>0$. In sum, we require

$$
\psi_{0}>0, \quad \psi_{1}>0, \quad \psi_{2}>0, \quad \operatorname{Pr}\left(Y<\frac{\psi_{1}}{-\rho} \mid Z\right)=1, \quad \psi_{2} c_{0}+\rho \psi_{1}>0
$$

This also implies that the optimal $s$ satisfies $0<s \leq \psi_{1} / c_{0}$. We again assume that the lower and upper boundary of $\mathcal{S}$ are not binding for the optimal $s$. It is not difficult to see that the expressions for the latter are the same as (7), with $s(z)$ now decreasing in $z$. The resulting expression for $V$ is also the same as in (8). We thus obtain,

Proposition 2. Consider the model with continuous effort, quadratic costs of effort, and the outcome function (6) with the conditions that ensure that it increases in effort and the treatment status. Then the exclusion restriction is violated iff $\rho^{2} \operatorname{var}(Z) /\left(2 c_{0}\right)>\gamma$.

This means, first of all, that the ER is likely to be violated if the treatment status and the effort before the treatment strongly interact in their effect on the outcome. This is because in that case the loss of choosing the wrong amount of effort is larger. Violation of the ER is more likely if $\gamma$ is small, which is trivial to understand.

It is also useful to discuss which model parameters do not affect the likelihood that the ER is violated. First, consider the parameter $\psi_{2}$. Until now we have not defined summary treatment effects yet. However, it is clear that any such measure depends on $\psi_{2}$. But this parameter does not affect the value of information. Therefore, the size of the (average) treatment effect does not affect the validity of the ER.

Secondly, consider the strength of the candidate instrument. This is usually defined by way of the strength of the association between $Z$ and $Y$, for example as measured by the correlation coefficient. This does not affect the validity of the ER at all. Thirdly, the validity of the ER does not depend on $\psi_{0}$ and $\psi_{1}$. The 
fact that it does not depend on the $\psi_{i}$ parameters reflects the fact that additive effects of treatment status and effort do not affect the ER (recall Proposition 1).

We now return to the related literature on decision making with a noisy signal about the state of the world. In this literature, agents receive a signal (say, $Z$ ) about the unknown state of the world (say, $Y$ ) and they have to decide on which action (say $s$ ) to take. The outcome (say, $f$ ) depends on $Y$ and $s$ (see Gollier, 2001, for a recent overview of models with signals, effort, and outcomes). The main difference with our setup is that here the focus is on the strength of the causal effect from the state of the world $Y$ on the signal $Z$ (or, equivalently, on the quality of the signal), whereas in our setup $Z$ causally affects $Y$. Also, this literature restricts attention to outcome functions $f$ that satisfy generalized notions of complementarity in the state of the world and the action of the individual (like supermodularity). Nevertheless, some of the results are directly applicable to our context.

Athey and Levin (2001) present a generalized version of the following result. Consider our model with continuous effort, where the treatment status $Y$ increases in $Z$ in the sense of first-order stochastic domination. The outcome function increases in effort and the treatment status, and the cross-derivative is positive. Then there is a monotone positive relation between $z$ and the optimal effort $s(z)$.

The general effect of the shape of the outcome function $(f)$ on the value of information $(V)$ is typically too hard to analyze in terms of the model primitives, if no parametric assumptions are made on $f$ (see Persico, 2000, and Athey and Levin, 2001). Perhaps most importantly, the results for given functions $s(z)$ emphasize the importance of the degree of complementarity of the outcome function on the value of information.

\subsection{Optimal behavior with discrete effort}

Now let $\mathcal{S}$ be discrete. For expositional reasons, we simplify the analysis by assuming that $s$ is binary, i.e. is taken from the set $\{0,1\}$. We adopt specification

(6) for $f$. The cost of effort function $c(s)$ is now represented by its two possible values $c(0)$ and $c(1)$. We define $c^{*}:=c(1)-c(0)$. This may be called the cost of effort. We may now simplify the expressions for the present values to

$$
\begin{aligned}
R(z) & =\psi_{0}-c(0)+\psi_{2} z+\max \left\{0, \psi_{1}+\rho z-c^{*}\right\} \\
R_{0} & =R(\bar{z})
\end{aligned}
$$


and the agent chooses $s=1$ iff the second term in the maximum exceeds 0 . It is immediately clear that for certain parameter values the optimal choice of $s$ is insensitive to small changes in the parameter values. To facilitate the exposition, we make the additional assumption that $Z$ has two possible values in the population of agents: $\operatorname{Pr}\left(Z=z_{1}\right)=p=1-\operatorname{Pr}\left(Z=z_{2}\right)$, with $z_{1} \neq z_{2}$ and $0<p<1$ and normalization $z_{1}>z_{2}$.

It is again useful to distinguish between the two cases in which treatment and effort are substitutes $(\rho<0)$ or complements $(\rho>0)$ for the outcome. This time we start with the former case, which requires again some restrictions on the range of values of the model parameters. By analogy to the previous subsection, we impose

$$
\psi_{0}>0, \quad \psi_{1}>0, \quad \psi_{2}>0, \quad \operatorname{Pr}\left(Y<\frac{\psi_{1}}{-\rho} \mid Z\right)=1, \quad \psi_{2}+\rho>0
$$

In fact, the results below also apply if $\psi_{2}+\rho=0$, meaning that with effort $s=1$ it is irrelevant whether the treatment is realized or not. This captures situations in which the outcome of interest is the transition from unemployment to work, if this is achieved with certainty by $s=1$ but treatment only increases the probability of a transition.

Equation (10) immediately implies that

$$
0 \leq s\left(z_{1}\right) \leq s_{0} \leq s\left(z_{2}\right) \leq 1
$$

As a result, we can distinguish between 4 "regimes", characterizing the optimal effort under different information sets for a given configuration of the model primitives:

- Regime 1. Always provide effort: $s\left(z_{1}\right)=s_{0}=s\left(z_{2}\right)=1$.

- Regime 2. Always provide effort except if it is known that $Z=z_{1}: 0=$ $s\left(z_{1}\right)<s_{0}=s\left(z_{2}\right)=1$.

- Regime 3. Only provide effort if it is known that $Z=z_{2}: 0=s\left(z_{1}\right)=s_{0}<$ $s\left(z_{2}\right)=1$.

- Regime 4. Never provide effort: $0=s\left(z_{1}\right)=s_{0}=s\left(z_{2}\right)$.

Using (10) the regimes can be characterized in terms of the model primitives,

- Regime 1 applies iff $\psi_{1}+\rho z_{1}>c^{*}$.

- Regime 2 applies iff $\psi_{1}+\rho z_{1} \leq c^{*}<\psi_{1}+\rho \bar{z}$. 
- Regime 3 applies iff $\psi_{1}+\rho \bar{z} \leq c^{*}<\psi_{1}+\rho z_{2}$.

- Regime 4 applies iff $\psi_{1}+\rho z_{2} \leq c^{*}$.

The intuition behind these results is exactly as in the previous subsection.

Next, we consider $V$. In Regime $1, V=0$, implying that the information is not bought. This is because the agent knows that he will always provide effort, under every policy, whether the policy is known or not, so the information is irrelevant for the optimal behavior. The same line of reasoning applies to Regime 4. So, for certain extreme parameter values, the agent does not acquire his value of $Z$. Now consider Regimes 2 and 3. We obtain that in Regime 2,

$$
V=p\left(c^{*}-\psi_{1}-\rho z_{1}\right)
$$

(recall that $p:=\operatorname{Pr}\left(Z=z_{1}\right)$ ). In this regime, the information on $Z$ is valuable if and only if in truth he has policy intensity $z_{1}$, because he does provide effort if he has no information. Therefore the value $V$ equals minus the expected loss of making such a wrong ${ }^{4}$ decision. More precisely, $V$ equals the product of [ the probability that, when $Z$ is not acquired, an effort $s_{0}$ is chosen that is not optimal in the light of the actual $Z$ ] and [ minus the loss of choosing $s_{0}$ given that the actual $Z$ would lead to another choice of $s$ ]. In Regime 2 , the statement in the first square brackets equals $\operatorname{Pr}\left(Z=z_{1}\right)$, which equals $p$. The statement in the second square brackets equals [ the additional cost $c^{*}$ of choosing $s_{0}=1$ compared to the costs one would have made if one would know that $\left.Z=z_{1}\right]$ minus [ the additional expected return of choosing $s_{0}=1$ compared to the expected return if one would know that $\left.Z=z_{1}\right]$.

Similarly, in Regime 3,

$$
V=(1-p)\left(\psi_{1}+\rho z_{2}-c^{*}\right)
$$

Note that $V>0$ if and only if the optimal effort when knowing that $Z=z_{1}$ differs from the optimal effort when knowing that $Z=z_{2}$. This is intuitively clear: only in these cases does the knowledge of $Z$ potentially have an effect on the effort provided.

Consider Figure 1, plotting $V$ against $c^{*}$. The maximum of $V$ as a function of $c^{*}$ is attained at the boundary between Regimes 2 and 3 , which means $c^{*}=$ $\psi_{1}+\rho \bar{z}$. This value $V_{\max }$ equals

\footnotetext{
${ }^{4}$ In this section, "wrong" is used in the sense of "wrong if the agent knows his actual value of $Z$ "'
} 


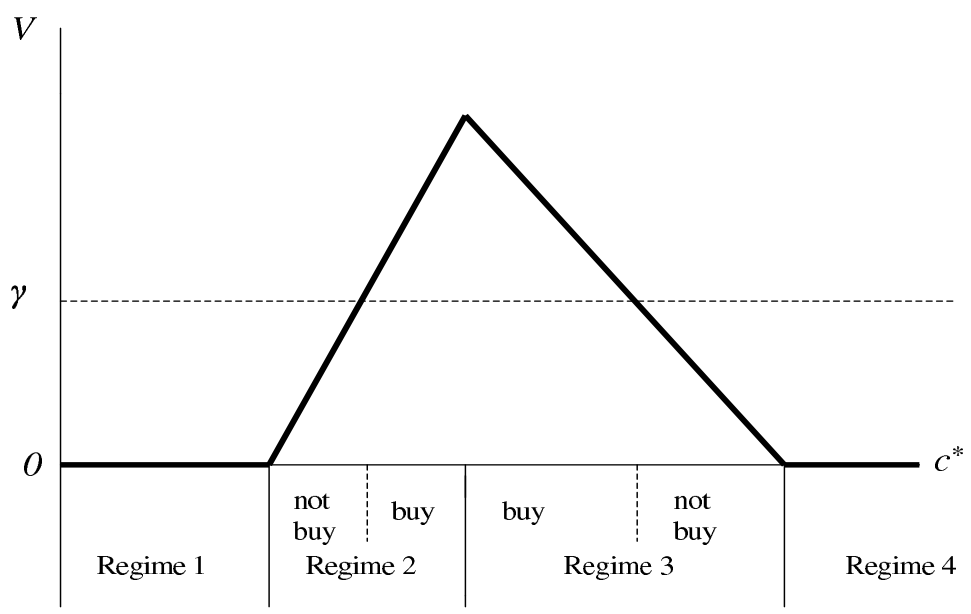

Figure 1. The value of information $V$ as a function (indicated by the fat solid curve) of the cost of effort $c^{*}:=c(l)-c(O)$.

Note: the parameter $\gamma$ denotes the information acquisition cost.

$$
V_{\max }=|\rho| p(1-p)\left(z_{1}-z_{2}\right)
$$

$V_{\max }>\gamma$ iff there are values $c(1), c(0)$ of the cost-of-effort function for which it is optimal to buy the information. We assume the latter because otherwise the analysis is irrelevant.

The expression for $V_{\max }$ is similar to the expression (8) for $V$ in the continuous case. In particular, note that $\operatorname{var}(Z)=p(1-p)\left(z_{1}-z_{2}\right)^{2}$. The comparative statics results concerning the effects of $\rho, \gamma$ and $\operatorname{var}(Z)$ for the continuous case therefore carry over to the present discrete case. For example, concerning $p$ we find that acquisition is more likely if $p$ is not too large and not too small. To understand this, note that $p=1 / 2$ maximizes the variance of $Z$ in the population, for given values of $z_{1}, z_{2}$. The a priori uncertainty concerning which value of $Z$ applies is largest for such intermediate values of $p$, and so is therefore the a priori probability of providing the wrong amount of effort.

What is particular about the discrete case is that for certain parameter values (namely in Regimes 1 and 4) the optimal $s$ does not depend on $Z$. Due to the continuity of the value functions, the ensueing low value of information also applies for parameter values such that one is in one of the other regimes but close to Regimes 1 and 4. In those cases the loss of making a wrong decision on $s$ is 
too small to justify the acquisition of one's value of $Z$. To analyze the associated comparative statics effects, one needs to examine for which parameter values one is likely to end up in Regimes 1 or 4 .

We find, first, that acquisition is more likely if $c^{*}$ is not too large and not too small, because acquisition takes place in Regimes 2 and 3, in particular around the value of $c^{*}$ at which these regimes border each other. To understand this result, note that for high or low values of $c^{*}$ the information does not lead to behavioral changes and so is useless.

The results for the case $\rho>0$ are a mirror image of those for $\rho<0$. The optimal efforts satisfy $0 \leq s\left(z_{2}\right) \leq s(\bar{z}) \leq s\left(z_{1}\right) \leq 1$. We can define Regimes 1-4 as follows: 1 . always provide effort, 2 . always provide effort except if it is known that $Z=z_{2}, 3$. only provide effort if it is known that $Z=z_{1}$, and 4. never provide effort. These can be characterized by: $1 . \psi_{1}+\rho z_{2}>c^{*}, 2$. $\psi_{1}+\rho z_{2} \leq c^{*}<\psi_{1}+\rho \bar{z}, 3 . \psi_{1}+\rho \bar{z} \leq c^{*}<\psi_{1}+\rho z_{1}$, and 4. $\psi_{1}+\rho z_{1} \leq c^{*}$. For sake of brevity we do not present the other results for this case.

One may combine the discrete case of this subsection with the continuous case of the previous subsection, e.g. by allowing the effort $s$ to attain all values in a fixed interval, e.g. $[0,1]$, with bounds that are binding for certain model parameter values. Sufficiently large parameter value changes then typically lead to results that correspond to those in this subsection.

\subsection{Examples}

In the leading example in this subsection, the treatment is participation in a job search assistance program by an unemployed individual, and one may think of the policy intensity as being different across the two geographical regions that are considered. For example, in one region, the budget for the program per potential participant may be larger, so that the individual probability of being treated is larger, holding everything else constant. An individual may not be aware of the variation in policy intensities, or he may be aware of the distribution of intensities but not know his personally relevant intensity, in which cases a regional dummy indicator may be a valid instrumental variable. Alternatively, $Z$ may be a deliberately randomized intention to treat, where the treatment concerns the increase in $Z$ from $z_{2}$ to $z_{1}$. In classical social experiments with full compliance, $z_{2}=0, z_{1}=1$, and $p=1 / 2$. In that case, the intention to treat, the policy intensity, and the treatment are all equivalent.

In the first leading example, an unemployed individual knows that there is a chance that he will be able to enroll in a job search assistance program, and 
this affects his job search strategy before actual enrollment. If the program is attractive and if the probability of enrollment into the program is known, then this probability affects the optimal private job search effort before enrollment into the program. Typically, the effort is lower if the probability of enrollment is higher, leading to a lower exit probability to work before enrollment, and this may lead to an under-estimation of the program effect on the employment rate say one year after inflow into unemployment.

We now apply the results in order to inquire the conditions under which $Z$ is a valid instrumental variable, that is, the conditions under which the agent does not acquire his value of $Z$.

To address the effects of the cost parameters $c(1)$ and $\gamma$ it is useful to slightly re-interpret the model as a model for a risk-averse agent who cannot transfer resources between time periods and who has a per-period utility function that displays decreasing absolute risk aversion. If the agent has a low per-period baseline income then the marginal utility losses of costs $c(1)$ in Period 2 and $\gamma$ in Period 1 are relatively high. The acquisition of information is unlikely for two reasons: it is expensive and it is useless because it does not affect optimal effort. The exclusion restriction is then relatively easy to justify. If the agent has a very high per-period baseline income then the marginal utility loss of costs $c(1)$ in Period 2 is small, and acquisition is again unlikely, but now only for the second of the above two reasons. ${ }^{5}$ Again, the exclusion restriction is then relatively easy to justify. For agents with per-period baseline incomes in between these extremes it is more likely that information on $Z$ is acquired, so then the exclusion restriction is more easily violated.

Of course, instead of interpreting the effects of the costs parameters in terms of marginal utility losses, one may also interpret them at face value. In that case, the exclusion restriction is less likely to be violated if the cost of providing effort has a rather high or low value and if the information cost is high.

Next, we find that the exclusion restriction is more likely to be violated if the candidate instrument covers a large shift in policy intensity and if it divides the population into groups of similar size. In the literature on instrumental variables, it is usually regarded to be attractive to have an instrument that has a strong effect on the treatment and that affects a substantial amount of agents. In our case this means that $z_{1}-z_{2}$ is large and that $p$ is close to $1 / 2$. From the above

\footnotetext{
${ }^{5}$ Unless of course the expected marginal return of providing effort is always very small as well. The problem with characterizing comparative statics effects on optimal behavior if the latter is a highly non-linear function of a large number of parameters is that there are often joint limiting values of a subset of parameters that "push" the result in any desired direction.
} 
it follows that this makes it more likely that information on $Z$ is acquired by the agent, so that the paradoxical conclusion follows that strong instruments are more likely to be invalid instruments.

The result on the effect of $p$ is especially relevant for social experiments where the randomized intention to treat is used as an instrumental variable. Typically, this intention to treat is randomized with probability equal to $1 / 2$. Our results suggest that (provided that the individual randomization outcome can only be acquired at a positive cost) it may be better to use a smaller or larger probability, because this reduces the likelihood that the agent has an incentive to acquire and use the value of the candidate instrumental variable. At the end of this section we return to such randomized intentions to treat.

We also find that the exclusion restriction is more likely to be violated if the outcome of interest strongly depends on interactions between the agent's effort before the outcome is realized on the one hand, and the agent's treatment status on the other. Also, it is less likely to be violated if all possible outcomes are very large or very small. ${ }^{6}$

So far we have only examined a single candidate instrumental variable. Clearly, if there are many such variables, each giving only limited information on the treatment assignment process, and if the cost of information acquisition is linear in the number of variables on which information is acquired, then this reduces the likelihood that the exclusion restriction is violated. Another obvious result concerns the timing of events: with a small amount of time between the moment at which the policy intensity is determined and the moment at which the treatment is realized, the scope for information acquisition is reduced.

We finish this section by some evaluating remarks about specific candidate instrumental variables in situations where there is a time span between the moment at which the policy intensity is determined and the moment at which the treatment is realized. First, as we just saw, a deliberately randomized intention to treat has as disadvantages that its realizations are typically available at no or low cost, and that the typical treatment probability of $1 / 2$ corresponds to a high incentive to acquire one's realization. Secondly, it is relatively common to use regional variation in the budget for (or, more generally, an indicator of the geographical availability of) active labor market programs as an instrumental

\footnotetext{
${ }^{6} \mathrm{By}$ definition, it is difficult to empirically test the above statements, because exclusion restrictions are untestable. One possibility would be to conduct a social experiment where the randomization probability for the policy intensity or the treatment probabilities vary between regions with otherwise identical conditions. Alternatively, one may attempt to observe agents' effort levels between the moments of policy intensity assignment and actual treatment.
} 
variable to study causal effects of the program. The present analysis suggests that this has the disadvantage that it is relatively easy for agents to learn the specific situation in their own region and the effect of this on the rate at which they may expect to be treated. Thirdly, instrumental variable analyses that restrict attention to agents with low or high resources (e.g. income) are more likely to be valid than analyses that include agents with intermediate resource levels.

\subsection{An economic model for selective non-compliance}

Suppose that agents can manipulate the probability distribution of their treatment status $Y$, by way of choosing an action $s$ before $Y$ is realized. As an extreme example, if the treatment status $Y$ is binary, they may switch treatment status by choosing an appropriate $s$. Agents' optimal $s$ may depend on $Z$, which may be acquired at a cost.

This model framework can be reformulated in terms of our framework. For convenience, we assume that $s$ does not affect the expected outcome function $f$, so we write $f:=f(Y)$ instead of $f(Y, s)$. At the same time, $s$ may affect the distribution of $Y \mid Z$, which we denote by $G(Y \mid Z ; s)$. If the agent does not acquire $Z$ then he chooses action or effort $s_{0}$ leading to $G\left(Y \mid Z ; s_{0}\right)$ which we denote by $H(Y \mid Z)$.

To see the connection to our framework, note that determining the optimal $s$ involves calculation of $\mathbb{E}_{Y \mid Z=z ; s} f(Y)$. This equals $\int f(y) d G(y \mid z ; s)$, which can be rewritten as

$$
\int f(y) d G(y \mid z ; s)=\int\left[\frac{f(y) d G(y \mid z ; s)}{d H(y \mid z)}\right] d H(y \mid z)
$$

The term in square brackets can now be interpreted as a new outcome function. If $z$ is a location parameter of the distribution of $Y \mid Z=z ; s$, so that $G(Y \mid z ; s)$ can be expressed as $G_{0}(Y-z \mid s)$ with $G_{0}$ functionally independent of $z$, then the above can be further simplified to

$$
\int f(y+z) d G_{0}(y \mid s)
$$




\section{The magnitude of the bias of the instrumental variable estimator if the exclusion restriction is violated}

\subsection{The parameter of interest and the estimator}

In this section we address under which circumstances the instrumental variable estimator is particularly heavily biased by the exclusion restriction violations that we consider. It is useful to return to the "reduced form" model representation from Section 2. IVE involves the estimation of the effect of $Y$ on $U$, holding all other determinants of $U$ constant. In the model, this is the partial derivative or first difference of $f$ with respect to its first argument. The classical IV regression estimator, if applied to data on $U, Y$, and $Z$, estimates $\operatorname{cov}(U, Z) / \operatorname{cov}(Y, Z)$. More precisely, estimation involves that these two covariances are replaced by their sample equivalents, and then the probability limit of the estimator, which we denote by $\widehat{\beta}_{I V}$, satisfies

$$
\widehat{\beta}_{I V} \rightarrow \frac{\operatorname{cov}(U, Z)}{\operatorname{cov}(Y, Z)}
$$

In our model framework, if the ER is valid, this equals

$$
\frac{\operatorname{cov}\left(f\left(Y, s_{0}\right), Z\right)}{\operatorname{cov}(Y, Z)}
$$

We simply define this to be the parameter of interest $\beta$. It captures the mean slope of the outcome as a function of treatment status for a given fixed effort. Note that this definition of $\beta$ is particularly sensible if $f$ is linear in $Y$, as is the case in specification (6), because then the slope of the outcome as a function of treatment status for a given fixed effort is a constant equal to $\beta$. Moreover, as we shall see, $\beta$ is a local average treatment effect if $Z$ is discrete with two points of support.

Expression (11) can be simplified by noting that $\operatorname{cov}(Y, Z)$ equals $\operatorname{var}(Z)$. Also, for any two random variables $X_{1}, X_{2}$, there holds that $\operatorname{cov}\left(X_{1}, X_{2}\right)=$ $\operatorname{cov}\left(\mathbb{E}\left(X_{1} \mid X_{2}\right), X_{2}\right)$. This results in

$$
\beta=\frac{\operatorname{cov}\left(\mathbb{E}\left(f\left(Y, s_{0}\right) \mid Z\right), Z\right)}{\operatorname{var}(Z)}
$$

If the ER does not apply, then 


$$
\widehat{\beta}_{I V} \rightarrow \frac{\operatorname{cov}(f(Y, s(Z)), Z)}{\operatorname{cov}(Y, Z)}=\frac{\operatorname{cov}(\mathbb{E}(f(Y, s(Z)) \mid Z), Z)}{\operatorname{var}(Z)}
$$

Note that $\widehat{\beta}_{I V}$ captures the over-all effect of $Z$ on the outcome. If the ER is violated then the over-all effect does not equal the causal treatment effect but also includes the causal chain that runs by way of the effort $s$. If $Z$ describes the assigned treatment as opposed to the actual treatment $Y$ then the over-all effect is usually called the intention-to-treat (ITT) effect on the outcome. This can be decomposed into the actual treatment effect $\beta$ and the announcement or ex ante effect of the treatment (see e.g. Abbring and Van den Berg, 2003, 2005, for this terminology). The latter thus equals $\widehat{\beta}_{I V}-\beta$, which is the asymptotic bias of the IV estimator $\widehat{\beta}_{I V}$ of $\beta$.

From an econometric regression point of view, one may state that the asymptotic bias of the IV estimator results from the fact that the size of the causal effect of treatment on outcome depends on the candidate IV. An alternative way to look at the asymptotic bias is to write the outcome equation as $U=$ $f\left(Y, s_{0}\right)+\left(f(Y, s(Z))-f\left(Y, s_{0}\right)\right)+\varepsilon$. By ignoring the dependence of $s$ on $Z$, an IV regression analysis takes the sum of the second and third terms in the right-hand side as the residual term in the outcome equation. Consequently, the candidate IV $Z$ is correlated to the error term in the outcome equation.

Note that instrumental variable estimators are typically biased in case of ER violations even if there is no selectivity in the treatment.

\subsection{Continuous effort}

Suppose that the ER is violated. We are first going to examine the asymptotic bias in the model with continuous effort, quadratic costs of effort, and the outcome function (6), with the conditions that ensure that it increases in effort and the treatment status. Subsequently we generalize the cost-of-effort function and the outcome function.

With the outcome function (6), $\beta$ as defined above is the average treatment effect in the population $\partial f(y, s) / \partial y$ which in this specific case does not depend on $y$, and which is evaluated at $s=s_{0}$. By substituting $f$ into (12), and substituting $s_{0}$, we obtain,

$$
\beta=\psi_{2}+\rho s_{0}=\psi_{2}+\rho \frac{\psi_{1}+\rho \bar{z}}{c_{0}}
$$

Similarly, by substituting (6) and $s(z)$ from (7) into (13), we obtain, 


$$
\widehat{\beta}_{I V} \rightarrow \psi_{2}+\frac{2 \rho \psi_{1}}{c_{0}}+\frac{\rho^{2}}{c_{0}} \frac{\operatorname{cov}\left(Z, Z^{2}\right)}{\operatorname{var}(Z)}
$$

As a result,

$$
\widehat{\beta}_{I V}-\beta \rightarrow \frac{\rho}{c_{0}}\left[\psi_{1}-\rho \bar{z}+\rho \frac{\operatorname{cov}\left(Z, Z^{2}\right)}{\operatorname{var}(Z)}\right]
$$

In the Appendix to this paper we prove the following:

Proposition 3. Consider the model with continuous effort, quadratic costs of effort, and the outcome function (6) with the conditions that ensure that it increases in effort and the treatment status. If the exclusion restriction is violated then the asymptotic bias of the IV estimator $\widehat{\beta}_{I V}$ has the same sign as $\rho$.

We use the monotonicity result of Athey and Levin (2001) (see Subsection 3.2) as an input in the derivation of results for more general model frameworks than considered so far in this subsection.

Proposition 4. Consider the model with continuous effort. Let the treatment status $Y$ increase in $Z$ in the sense of first-order stochastic domination. Let the outcome function increase in effort and treatment status, and suppose that the cross-derivative is positive. If the exclusion restriction is violated then the asymptotic bias of the IV estimator $\widehat{\beta}_{I V}$ is positive.

\subsection{Discrete effort}

Let $s$ be binary and let $Z$ have a discrete distribution with points of support $z_{1}>z_{2}$, like in Subsection 3.3. It can be shown that $\beta$ in (12) then simplifies to

$$
\beta=\frac{\mathbb{E}\left(f\left(Y, s_{0}\right) \mid z_{1}\right)-\mathbb{E}\left(f\left(Y, s_{0}\right) \mid z_{2}\right)}{z_{1}-z_{2}}
$$

which is a local average treatment effect (compare Imbens and Angrist, 1994; note that the denominator equals $\left.\mathbb{E}\left(Y \mid z_{1}\right)-\mathbb{E}\left(Y \mid z_{2}\right)\right)$. If the $\mathrm{ER}$ is valid then the IV estimator $\widehat{\beta}_{I V}$ converges to this number. Accordingly, we can use a Wald estimator as IV estimator.

If the ER is violated then we can simplify (13) to

$$
\widehat{\beta}_{I V} \rightarrow \frac{\mathbb{E}\left(f\left(Y, s\left(z_{1}\right)\right) \mid z_{1}\right)-\mathbb{E}\left(f\left(Y, s\left(z_{2}\right)\right) \mid z_{2}\right)}{z_{1}-z_{2}}
$$

so that again $\widehat{\beta}_{I V}$ captures the over-all effect of $Z$ on the outcome. 
Now let us proceed by taking the outcome function $f$ to satisfy (6). From the above (as well as from the previous subsection) it immediately follows that $\beta=\psi_{2}+\rho s_{0}$. If the ER is violated then necessarily $s\left(z_{1}\right) \neq s\left(z_{2}\right)$. With $\rho<0$, violation implies that $0=s\left(z_{1}\right)<s\left(z_{2}\right)=1$, whereas with $\rho>0$, this is reversed. By elaborating on equation (13) or on equation (14), we obtain

$$
\begin{array}{ll}
\widehat{\beta}_{I V} \rightarrow \psi_{2}-\frac{\psi_{1}+\rho z_{2}}{z_{1}-z_{2}} & \text { if } \rho<0 \\
\widehat{\beta}_{I V} \rightarrow \psi_{2}+\frac{\psi_{1}+\rho z_{1}}{z_{1}-z_{2}} & \text { if } \rho>0
\end{array}
$$

Depending on the sign of $\rho$ and the value of $s_{0}$, we have four different expressions for the asymptotic bias,

$$
\begin{array}{cc}
\widehat{\beta}_{I V}-\beta \rightarrow-\frac{\psi_{1}+\rho z_{2}}{z_{1}-z_{2}} & \text { if } \rho<0 \text { and } s_{0}=0 \\
\widehat{\beta}_{I V}-\beta \rightarrow-\frac{\psi_{1}+\rho z_{1}}{z_{1}-z_{2}} & \text { if } \rho<0 \text { and } s_{0}=1 \\
\widehat{\beta}_{I V}-\beta \rightarrow \frac{\psi_{1}+\rho z_{1}}{z_{1}-z_{2}} & \text { if } \rho>0 \text { and } s_{0}=0 \\
\widehat{\beta}_{I V}-\beta \rightarrow \frac{\psi_{1}+\rho z_{2}}{z_{1}-z_{2}} & \text { if } \rho>0 \text { and } s_{0}=1
\end{array}
$$

Thus,

Proposition 5. Consider the model with binary effort, a binary candidate IV, and the outcome function (6) with the conditions that ensure that it increases in effort and the treatment status. If the exclusion restriction is violated then the asymptotic bias of the $I V$ estimator $\widehat{\beta}_{I V}$ has the same sign as $\rho$.

The bias terms are larger if $z_{1}$ and $z_{2}$ are close. However, recall that this result is derived under the simplifying assumptions that all agents are in Regime 2 and acquire information on $Z$, so that $z_{1}$ and $z_{2}$ can not be too close.

From the interpretation of the bias as capturing the announcement effect or ex ante effect of the treatment, it follows that a large bias term is equivalent to a large ex ante effect. This means that a large bias is often associated to a high value of information.

Suppose the circumstances are such that it is plausible that the exclusion restriction is violated, so IV cannot be applied. One way to proceed is to estimate a structural economic model. Alternatively, if the model framework is dynamic with sufficient variation in the timing of treatment and the outcome of interest, 
then one may follow the so-called "timing-of-events" approach (Abbring and Van den Berg, 2003), that is, impose some semi-parametric structure and exploit the variation in the timing of events for identification of a causal treatment effect. Note that the value of $Z$, if observed, does not have any influence on optimal behavior after the actual treatment. Abbring and Van den Berg (2005) exploit this so-called "ex post exclusion restriction" for identification of selection effects. They also demonstrate that, with sufficient semi-parametric structure, the information in the timing of events enables identification of the ex ante effect and the treatment effect. It is a topic for further research to investigate under which conditions the observation of indicators of the agent's effort level before treatment provides useful additional information.

Of course, exclusion restrictions for instrumental variable estimation may break down for other reasons than those considered in this paper. Notably, the agent's value of the candidate instrument may be affected by unobserved characteristics of the agent that have a direct causal effect on the outcome variable. This is prevented if the candidate instrument is the result of a deliberate randomization, like a deliberately randomized intention-to-treat variable in a social experiment.

\section{Conclusions}

Exclusion restrictions for instrumental variable estimation are untestable and therefore need to be justified externally. We consider situations in which there is a time interval between the moment the agent realizes that he may be exposed to the policy and the actual exposure. We economically analyze the decision whether to acquire information concerning the value of the candidate instrumental variable.

The results suggest, first, that the exclusion restriction is more likely to be violated if the candidate instrument covers a large shift in policy intensity or if it divides the population into groups of similar size. We also find that the exclusion restriction is more likely to be violated if the outcome of interest strongly depends on interactions between the agent's effort before the outcome is realized on the one hand, and the agent's treatment status on the other.

Deliberate randomization of the intention to treat, like in the case of social experiments, does not help. The randomization outcome is typically available to the agent at low cost, and the typical randomization probability of $1 / 2$ corresponds to a high incentive to acquire one's realization. In fact, it may be better to use a smaller or larger probability, because this reduces the incentive to acquire 
and use the value of the candidate instrumental variable.

Having a weak instrument does not help either. Weakness of the candidate instrument, as defined or measured in ways proposed in the literature, is not directly related to the likelihood that the exclusion restriction is violated.

With discrete effort, instrumental variable analyses that restrict attention to agents with low or high resources (e.g. income) are more likely to be valid than analyses that include agents with intermediate resource levels. The reason is that for the former groups, the information is useless because it does not affect optimal effort. In addition, for the low resource agents, it may be too expensive.

Finally, concerning the bias in case of violation of the exclusion restriction, we find that typically, it is large if the value of information is large. 


\section{References}

Abbring, J.H. and G.J. van den Berg (2003), "The non-parametric identification of treatment effects in duration models", Econometrica, 71, 1491-1517.

Abbring, J.H. and G.J. van den Berg (2005), "Social experiments and instrumental variables with duration outcomes", Working paper, Free University Amsterdam, Amsterdam.

Angrist, J.D., G.W. Imbens, and D.B. Rubin (1996), "Identification of causal effects using instrumental variables", Journal of the American Statistical Association, 91, 444-455.

Athey, S. and J. Levin (2001), "The value of information in monotone decision problems", Working paper, Stanford University, Stanford.

Blundell, R. and T. MaCurdy (1999), "Labor supply", in O. Ashenfelter and D. Card, editors, Handbook of Labor Economics, Volume III, North-Holland, Amsterdam.

Collins, H.M. and T. Pinch (1998), The golem at large, Cambridge University Press, Cambridge, UK.

Gollier, C. (2001), The Economics of Risk and Time, MIT Press, Cambridge.

Heckman, J.J., R.J. LaLonde, and J.A. Smith (1999), "The economics and econometrics of active labor market programs", in O. Ashenfelter and D. Card, editors, Handbook of Labor Economics, Volume III, North-Holland, Amsterdam.

Heckman, J.J. and S. Navarro (2005), "Dynamic discrete choice and dynamic treatment effects", Working paper, University of Chicago, Chicago.

Imbens, G.W. and J.D. Angrist (1994), "Identification and estimation of local average treatment effects", Econometrica, 62, 467-475.

Imbens, G.W. and W.K. Newey (2002), "Identification and estimation of triangular simultaneous equation models without additivity", Working paper, M.I.T., Cambridge.

Persico, N. (2000), "Information acquisition in auctions", Econometrica, 68, 135148.

Schuklenk, U. (2003), "AIDS: bioethics and public policy", New Review of Bioethics, $1,127-144$.

Shohat, J.A. and J.D. Tamarkin (1943), The Problem of Moments, American Mathematical Society, New York. 
Stock, J.H., J.H. Wright, and M. Yogo (2002), "A survey of weak instruments and weak identification in generalized method of moments", Journal of Business and Economic Statistics, 20, 518-529. 